\title{
THE ORIGIN OF SOFT X-RAYS IN DQ HERCULIS
}

\author{
K. MuKaI ${ }^{1,2}$ AND M. STILL ${ }^{2}$ \\ NASA Goddard Space Flight Center, Code 662, Greenbelt, MD 20771
}

AND

F. A. RingWALD

Department of Physics, California State University at Fresno, 2345 East San Ramon Avenue, MS MH37, Fresno, CA 93740-8031 Received 2002 October 13; accepted 2003 May 5

\begin{abstract}
DQ Herculis (Nova Herculis 1934) is a deeply eclipsing cataclysmic variable containing a magnetic white dwarf primary. The accretion disk is thought to block our line of sight to the white dwarf at all orbital phases because of its extreme inclination angle. Nevertheless, soft X-rays were detected from DQ Her with the ROSAT PSPC. To probe the origin of these soft X-rays, we have performed Chandra ACIS observations. We confirm that DQ Her is an X-ray source. The bulk of the X-rays are from a pointlike source and exhibit a shallow partial eclipse. We interpret this as being due to scattering of the unseen central X-ray source, probably in an accretion disk wind. At the same time, we observe what appear to be weak extended X-ray features around DQ Her, which we interpret as an X-ray-emitting knot in the nova shell.
\end{abstract}

Subject headings: binaries: eclipsing — novae, cataclysmic variables — stars: individual (DQ Herculis) — X-rays: stars

\section{INTRODUCTION}

A classical nova eruption (see Shara 1989 for a review) is a thermonuclear runaway in a cataclysmic variable $(\mathrm{CV}$; a semidetached binary in which a white dwarf primary accretes from a late-type, usually $\mathrm{M}$ dwarf, secondary). The runaway is caused when $\sim 10^{-4} M_{\odot}$ of hydrogen-rich gas is accreted onto the white dwarf surface; this material becomes degenerate, until the pressure and temperature at the base of this envelope start the runaway reaction. A nova eruption ejects most of the accreted envelope. After a poorly known recurrence time $\left(10^{3}-10^{5} \mathrm{yr}\right)$, the underlying binary accumulates enough fresh fuel to erupt again. Novae are of interest to nuclear astrophysicists, they are useful as extragalactic distance indicators, they play a role in Galactic chemical evolution, and they are likely sources of $\gamma$-rays. To understand a nova, it is essential to study the underlying system away from its eruption. Conversely, the study of nova eruptions is essential to understanding the secular evolution of CVs.

The subject of this paper, DQ Herculis (Nova Herculis 1934), has played a key historical role in the studies of nova eruptions and of postnova binaries (hereafter old novae) because of its brightness and fortuitous viewing geometry. The 1934 classical nova eruption of DQ Her reached $m_{v} \sim 1.3$ and enabled extensive observations (see Martin 1989 for a review). The discovery of eclipses in DQ Her (Walker 1956) was pivotal in establishing the standard picture of CVs. Walker (1956) also discovered the coherent $71 \mathrm{~s}$ photometric oscillation; we now understand that DQ Her contains a magnetic white dwarf, accreting onto the magnetic polar regions, that are offset from the rotational poles, i.e., an oblique rotator. The ejecta of the 1934 eruption are visible in $\mathrm{H} \alpha$ and other emission lines as an elliptical shell:

${ }^{1}$ Columbia Astrophysics Laboratory, Columbia University, 550 West 120th Street, New York, NY 10027.

${ }^{2}$ Universities Space Research Association. as of 1978, $43 \mathrm{yr}$ after the eruption, the equatorial and polar radii (expansion velocities) of the DQ Her shell were $6.3 \pm 0$ ".2 $\left(384 \pm 21 \mathrm{~km} \mathrm{~s}^{-1}\right)$ and $8 . \prime 6 \pm 0.2(528 \pm 25 \mathrm{~km}$ $\mathrm{s}^{-1}$ ), respectively (Herbig \& Smak 1992). Herbig \& Smak (1992) found no evidence for a deceleration of the expansion and derived a revised distance estimate of $561 \pm 19 \mathrm{pc}$ (we use this value throughout this paper). This makes DQ Her among the nearest old novae known. By 1995 September (61 $\mathrm{yr}$ after eruption), the equatorial and polar radii have grown to 9 ". $1 \pm 0$ ".8 and 12 ". $5 \pm 0$ ".8, respectively, still consistent with no deceleration, according to our analysis of an archival Hubble Space Telescope WFPC2 image.

The eclipsing nature of DQ Her in principle allows us to place tight constraints on its system parameters. The optical eclipse width, measured from midingress to midegress, as is customary, is $0.110 \pm 0.003$ cycles (as compiled from literature by Horne, Welsh, \& Wade 1993). Assuming that the source of eclipsed light is centered on the white dwarf, this can be used to constrain the relationship between the mass ratio $q=M_{2} / M_{1}$ (where $M_{1}$ and $M_{2}$ denote the masses of the white dwarf and the mass donor, respectively) and the inclination angle $i$. Horne et al. (1993) measured the radial velocity amplitude of the secondary $K_{2}$ through the detection of the $\mathrm{Na}$ I infrared doublet absorption lines. This, combined with the eclipse width and the $K_{1}$ from literature, has led to their estimates of $q=0.66 \pm 0.04, i=86.5 \pm 1.6$, $M_{1}=0.60 \pm 0.07 M_{\odot}$, and $M_{2}=0.40 \pm 0.05 M_{\odot}$.

About $20 \mathrm{X}$-ray-bright CVs with coherent spin modulations have been discovered since the late 1970s and are classified as intermediate polars (IPs), or DQ Her stars (Patterson 1994). They are among the brightest CVs in the $2-10 \mathrm{keV}$ band, with luminosities generally in excess of $10^{32}$ ergs s${ }^{-1}$. However, the prototype DQ Her was not detected in an Einstein Imaging Proportional Counter (IPC) observation with a $2 \sigma$ upper limit of 0.0046 counts s ${ }^{-1}$ (Córdova, Mason, \& Nelson 1981). One of the hypotheses of Córdova et al. (1981), that high inclination may be the cause of this nondetection, found support in a model of the phase shifts 
of the $71 \mathrm{~s}$ oscillation during eclipse (Petterson 1980). In this model, a beam of high-energy photons originating on the white dwarf is reprocessed by the accretion disk and results in the optical oscillation. Petterson (1980) finds a good fit at $i \sim 89^{\circ}$, although the actual value of $i$ can be lower, as long as the entire front half of the disk including the white dwarf is obscured by the outer edge of the disk. Such permanent obscuration of the white dwarf would greatly reduce the $\mathrm{X}$-rays reaching Earth.

Despite the high inclination angle, X-rays were detected from DQ Her with the ROSAT Position Sensitive Proportional Counter (PSPC; Silber et al. 1996). ${ }^{3}$ The inferred luminosity (0.1-2.0 keV) of $\sim 4.5 \times 10^{30} \mathrm{ergs} \mathrm{s}^{-1}$ is modest for an IP. We note that, using a thermal plasma model with $k T=0.3 \mathrm{keV}$ and $N_{\mathrm{H}}=1.0 \times 10^{20} \mathrm{~cm}^{-2}$, consistent with the PSPC spectrum, and the revised distance of $561 \mathrm{pc}$ (both are different from the values assumed by Córdova et al. 1981), the Einstein IPC upper limit corresponds to $2.8 \times 10^{30}$ ergs s${ }^{-1}$ in the IPC band $(0.4-4 \mathrm{keV})$ or $3.3 \times 10^{30} \mathrm{ergs} \mathrm{s}^{-1}$ in the $0.1-2.0 \mathrm{keV}$ band. These numbers may reflect source variability or the limit of accuracy of cross-instrument comparisons; neither would be surprising. A deep eclipse is not observed, consistent with the permanent obscuration of the white dwarf, suggesting an alternative origin of the observed X-rays. Although Silber et al. (1996) considered a likely origin to be the secondary, it has to be emitting X-rays at significantly above the saturation limit $\left(10^{-3} L_{\mathrm{bol}}\right.$, or a few times $10^{29} \mathrm{ergs} \mathrm{s}^{-1}$ in this case) seen in rapidly rotating latetype dwarfs (see, e.g., Singh et al. 1999). If not the secondary, what is the origin of the soft X-rays observed from DQ Her?

Only $\sim 150$ photons were detected in the ROSAT observation, insufficient for further progress. We have therefore secured a Chandra X-Ray Observatory Advanced CCD Imaging Spectrometer (ACIS) observation of DQ Her. We describe the observation and data reduction in $\S 2$, present the results in $\S 3$, and discuss the implications in $\S 4$.

\section{OBSERVATIONS}

We observed DQ Her with the Chandra (Weisskopf, O'Dell, \& van Speybroeck 1996) ACIS without a grating, placing the object on the back-illuminated S3 chip. ${ }^{4}$ The observations were performed as two pointings, one from 2001 July 26 13:00 UT to July $2702: 31$ UT and the other from 2001 July 29 17:09 UT to July 30 02:27 UT, for a total integration time of $68.8 \mathrm{ks}$. These observations were performed at near-identical pointing positions and roll angles. We took the outputs from the processing pipeline at the Chandra X-Ray Center and extracted images, spectra, and light curves from these observations individually, before combining the results.

We detect a source at R.A. $=18^{\mathrm{h}} 07^{\mathrm{m}} 30^{\mathrm{s}} \cdot 24$, decl. $=$ $+45^{\mathrm{d}} 51^{\mathrm{m}} 32^{\mathrm{s}} .6(\mathrm{~J} 2000.0)$ using the CIAO routine "celldetect." Although this is offset by 1".7 from the position of DQ Her given in Downes et al. (2001), this is likely to reflect the limitation of comparison of positions measured using different astrometric reference frames (R. A. Downes 2002,

\footnotetext{
${ }^{3}$ Patterson (1994) was already aware of this detection, citing Patterson $\&$ Eracleous (1994, in preparation).

${ }^{4}$ For details of the ACIS instrument, see the Web site at http://www.astro.psu.edu/xray/acis.
}

private communication). We identify this X-ray source with DQ Her based on this positional coincidence and on the orbital variability (see $\S 3.2$ ).

We extracted source spectra and light curves from a $5^{\prime \prime}$ radius circular region and background from an annulus with a $40^{\prime \prime}$ outer radius and a $20^{\prime \prime}$ inner radius centered on the source. The size of the background annulus is chosen to exclude the nova shell emission (see $\S 3.3$ ). The source count rate is 0.025 counts s$~^{-1}\left(3 \times 10^{30} \mathrm{ergs} \mathrm{s}^{-1}\right.$ in the $0.2-5 \mathrm{keV}$ band, based on the spectral model discussed below), while the background in the source region (scaled from the counts in the background region by the detector areas) is estimated to be $8.2 \times 10^{-4}$ counts s $^{-1}$. DQ Her did not show a significant difference in count rate between the two observations.

\section{RESULTS}

\subsection{Spectral Characteristics}

There is a bump in the observed ACIS-S spectrum of DQ Her around $1 \mathrm{keV}$ (left-hand panel of Fig. 1) that is not in the instrument response. Because of this, the spectrum cannot be fitted with smooth continuum models (power-law, bremsstrahlung, or blackbody) alone. Using models of optically thin thermal plasma emission, such as MEKAL (Mewe, Gronenschild, \& van den Oord 1985; Mewe, Lemen, \& van den Oord 1986; Liedahl, Osterheld, \& Goldstein 1995), the $1 \mathrm{keV}$ bump is interpreted as a complex of lines in the $0.6-1.2 \mathrm{keV}$ range, notably lines of $\mathrm{O}$ VIII $(0.64$ $\mathrm{keV}), \mathrm{Fe}$ XVII $(0.73$ and $0.83 \mathrm{keV})$, and $\mathrm{Ne}$ IX $(0.90 \mathrm{keV})$. These lines, however, are weaker relative to the continuum than the predictions of single-temperature plasma models. Moreover, the continuum above $2 \mathrm{keV}$ is much stronger than expected for plasma temperatures that give rise to these lines $(k T \sim 0.6 \mathrm{keV})$. As a result, a single-component MEKAL model fit also fails to reproduce the observed spectrum.

The simplest successful representation $\left(\chi_{\nu}^{2}=1.4\right.$; see Fig. 1) is a two-component model ${ }^{5}$ consisting of a $k T=$ $0.59 \pm 0.04 \mathrm{keV}$ MEKAL plasma model plus a power law of photon index $2.7 \pm 0.2$, with $N_{\mathrm{H}}=(2.9 \pm 1.2) \times 10^{20} \mathrm{~cm}^{-2}$. The power-law component in our model provides the added continuum necessary to achieve an acceptable fit, both near the lines $(0.8-1.2 \mathrm{keV})$ and at higher energies $(2-5 \mathrm{keV})$. Inferred intrinsic flux in the $0.2-5 \mathrm{keV}$ band is $8.2 \times 10^{-14}$ ergs $\mathrm{cm}^{-2} \mathrm{~s}^{-1}$, or a luminosity of $3.1 \times 10^{30} \mathrm{ergs} \mathrm{s}^{-1}$. DQ Her was not significantly detected above $5.0 \mathrm{keV}$ [we obtain a count rate and an estimated $1 \sigma$ error in the $5-8 \mathrm{keV}$ band of $(0.3 \pm 2.0) \times 10^{-4}$ counts $\mathrm{s}^{-1}$ ], although this is in part due to the lower effective area of the Chandra ACIS instrument at high energies. We take $2.0 \times 10^{-4}$ counts s$^{-1}$ as our upper limit, which corresponds to $4 \times 10^{29} \mathrm{ergs} \mathrm{s}^{-1}$, assuming a power-law spectral shape with a photon index of 2 and a source distance of $561 \mathrm{pc}$.

Note, however, that our adopted model for the spectrum is not unique. Given the modest number of detected photons $(\sim 1700)$ and instrumental limitations (in both bandpass and spectral resolution), there can be a wide range of models that fit the data. We are only confident that DQ Her shows a spectral feature around $1 \mathrm{keV}$, which mimics a

\footnotetext{
${ }^{5}$ We have also included the ACISABS model in the fit, to account for instrumental absorption due to contaminant, at the appropriate level.
} 

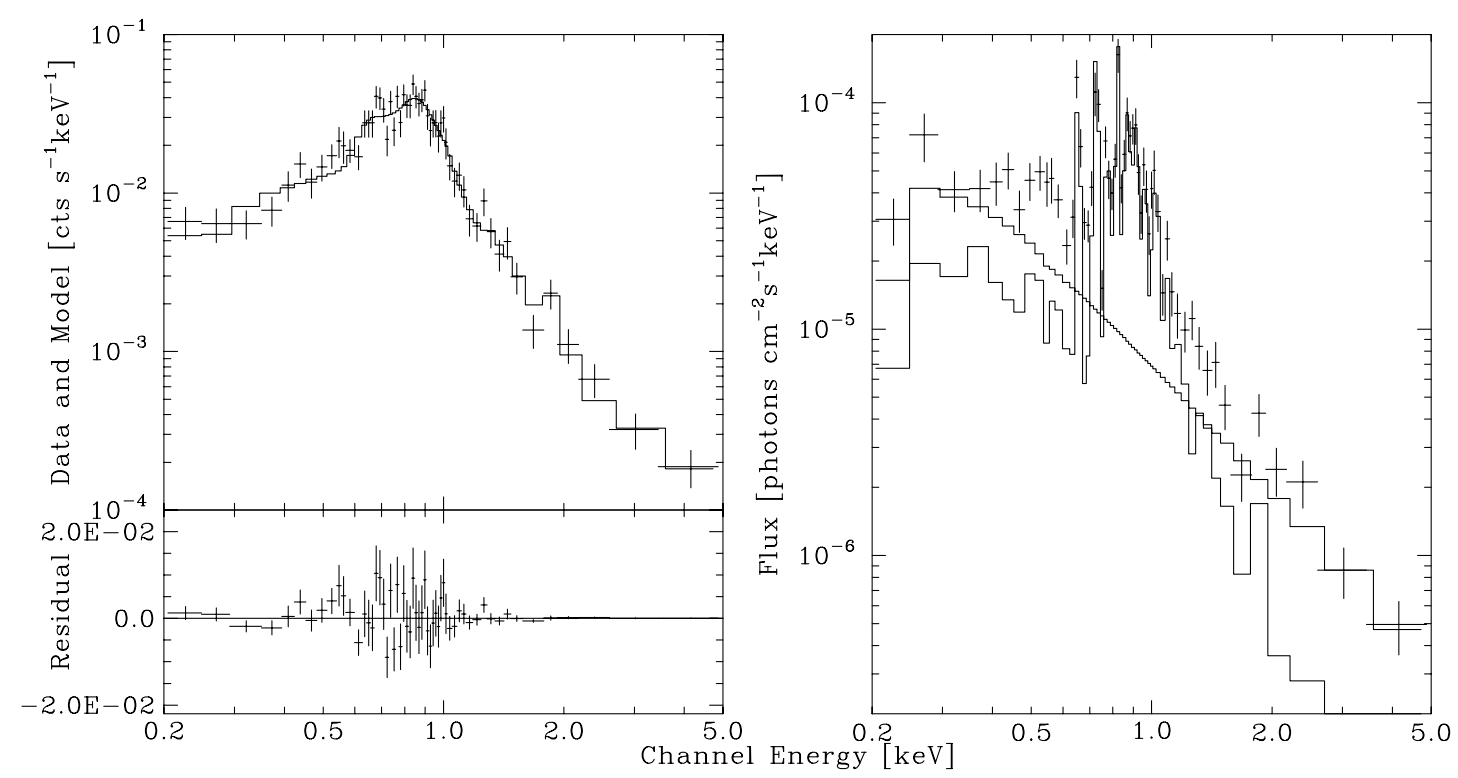

FIG. 1.-Chandra ACIS spectrum of DQ Her. Left: The observed spectrum is shown in the top panel, with the best-fit two-component model folded through the instrument response, while the residuals are shown in the bottom panel. Right: The photon spectrum inferred from the fit, shown with the power-law and thin thermal plasma components.

collisionally excited, optically thin plasma of $k T \sim 0.6 \mathrm{keV}$. The presence of such a plasma would also lead to a Si line at $1.85 \mathrm{keV}$, which may also be present in the data. Consequently, spectral modeling alone of the Chandra data is insufficient to uncover the origin of the soft X-rays in DQ Her.

\subsection{Timing Characteristics}

We show, in Figure 2, the ACIS light curve of DQ Her folded on the orbital ephemeris of Zhang et al. (1995) into 16 bins per cycle. The ephemeris has an estimated accuracy of $\pm 11 \mathrm{~s}$ for this epoch (see $\S 4.1$ for further details). A partial eclipse, lasting for about 3 bins and about $30 \%$ deep, is clearly seen. In this representation, the X-ray eclipse center appears to be offset from the ephemeris prediction by onehalf of the bin size, i.e., 0.03 cycles, or 500 s. Moreover, the $\mathrm{X}$-ray eclipse appears to be wider ( 3 bins, or 0.1875 cycles)

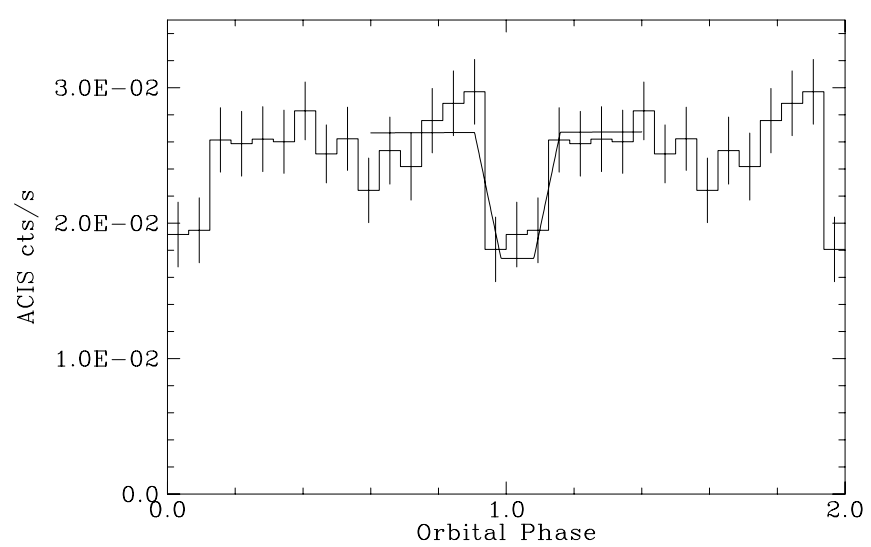

FIG. 2.-Chandra ACIS light curve of DQ Her, folded on the orbital period and plotted twice. Each bin is $1 / 16$ of the orbital period. Also shown is a piecewise linear model of the eclipse; the parameters are from the best-fit model to the 32 bin per cycle version of the folded light curve. than the optical eclipse $(0.110 \pm 0.003$, according to a compilation in Horne et al. 1993), which should be 2 bins in Figure 2.

To quantify this impression, we have fitted the 32 bin per cycle version of the light curve using a piecewise linear function with symmetric ingress and egress; we show our best-fit model in Figure 2. The eclipse center is found to be at phase $0.033_{-0.027}^{+0.030}(90 \%$ confidence), confirming the offset. However, the eclipse width (midingress to midegress) is measured to be $0.18_{-0.12}^{+0.05}(90 \%)$ cycles; thus, we cannot claim that the $\mathrm{X}$-ray eclipse is definitely wider than in the optical. In addition to the formal uncertainties, there is additional uncertainty arising from our choice of a specific model; thus, both these numbers need confirmation. Our Chandra data are of insufficient quality for us to be able to derive further details of the eclipse profile, such as ingress/egress durations.

\subsection{Imaging Characteristics}

At first glance, the Chandra ACIS image of DQ Her looks like that of a point source. Indeed, over $70 \%$ of the flux is contained within $1^{\prime \prime}\left(\sim 560 \mathrm{AU}\right.$, or $\left.8.4 \times 10^{15} \mathrm{~cm}\right)$ of the image centroid, as expected for a soft point source. However, a closer inspection of the image shows that there is a region about $2^{\prime \prime} \times 2^{\prime \prime}$ in area and about $10^{\prime \prime}$ northeast of the point source with more photons (28) than are expected by chance $(\sim 8)$. These excess counts appear equally distributed over this area, and they do not display the sharply peaked core of the point-spread function (PSF) expected of a point source.

We have investigated this further by constructing radial profiles of the ACIS image of DQ Her in several energy bands. We then fit a model consisting of a flat background plus the PSF available in the Chandra calibration database (interpolated in energy and off-axis angle as appropriate). We find notable excesses in the azimuthally averaged radial profiles in the $0.2-0.5$ and $0.5-0.8 \mathrm{keV}$ bands (Fig. 3; we find no significant features at energies above $0.8 \mathrm{keV}$, although this may in part be due to poor counting statistics). The 


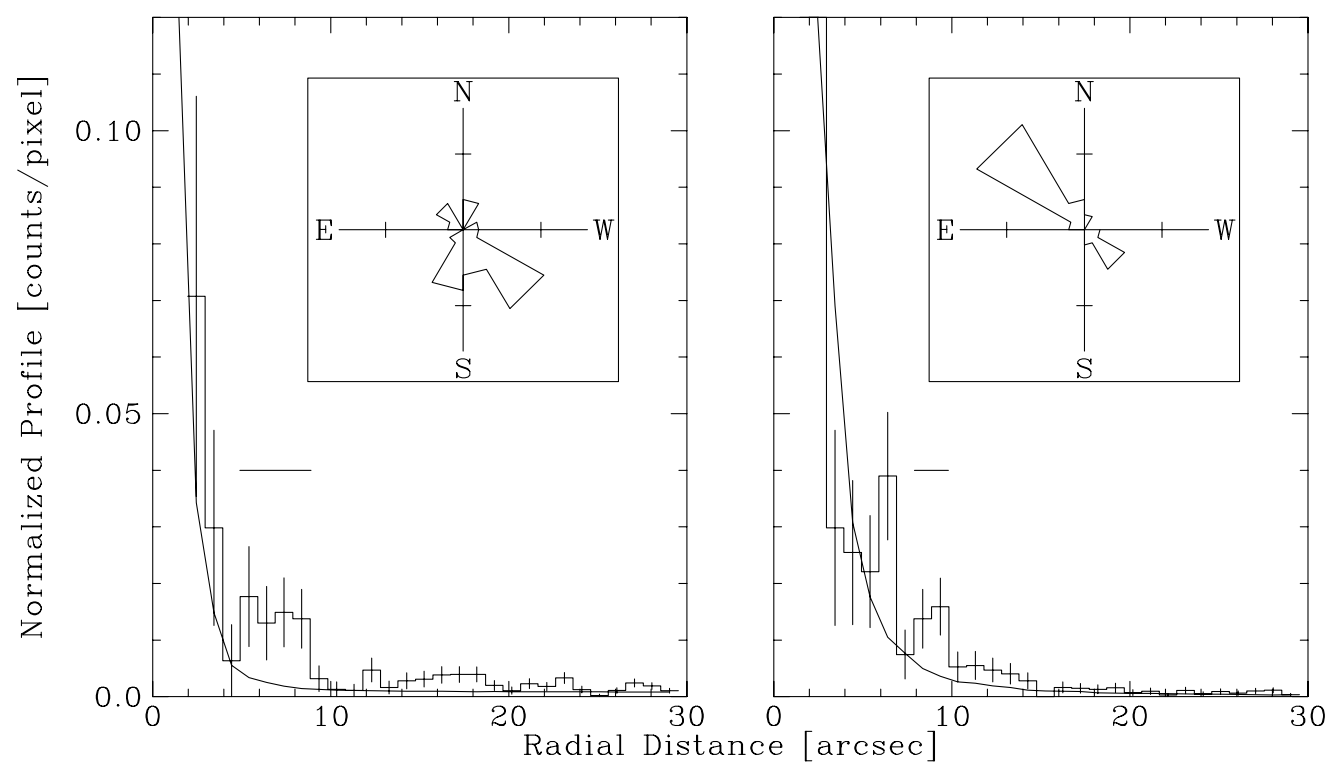

FIG. 3.-Radial profiles of the Chandra ACIS-S X-ray image of DQ Her in the $0.2-0.5 \mathrm{keV}($ left $)$ and $0.5-0.8 \mathrm{keV}$ (right) bands. The data are plotted as a histogram with errors, while the best-fit model is plotted as a solid line. The central $\sim 1^{\prime \prime}$ determines the normalization of the profile, but has been omitted from this figure to make the wings of the PSF visible. The horizontal bars indicate the two prominent features examined further in the insets. They are histograms in polar coordinates of the excess counts (see text for details). The tick marks indicate 5 counts per bin.

statistical significance of this is unfortunately difficult to establish: our experiences with other bright sources is that the PSF fit is usually less than perfect because of effects of pile-up, background nonuniformity, uncertainties in the point-source location, and imperfect calibration of the PSF. However, the structures (clusters of several points above the best-fit models) seen in the radial profiles of DQ Her are unusual.

The two features marked with horizontal bars (one at $5^{\prime \prime}-$ $9^{\prime \prime}$ in the $0.2-0.5 \mathrm{keV}$ band and another at $8^{\prime \prime}-10^{\prime \prime}$ in the $0.5-$ $0.8 \mathrm{keV}$ band) contain $18.0 \pm 4.6$ and $12.2 \pm 4.1$ counts, respectively. Other possible features either have too few photons (the $6^{\prime \prime}-7^{\prime \prime}$ bin in the $0.5-0.8 \mathrm{keV}$ profile has 5) or are outside the optically detected nova shell (the $12^{\prime \prime}-20^{\prime \prime}$ bin in the $0.2-0.5 \mathrm{keV}$ profile) and will not be discussed further. For the two features, we also construct an azimuthal histogram in $30^{\circ}$ bins of photons falling within the appropriate radial and energy bins. We plot this in the insets of Figure 3 as a histogram in polar coordinates (the position angle on the graph corresponds to the position angle on the sky, and radial distance from the center is proportional to the number of photons in that bin). We find that the $0.2-0.5 \mathrm{keV}$ feature is about $8^{\prime \prime}$ south-southwest of DQ Her, while the $0.5-0.8 \mathrm{keV}$ feature is about $9^{\prime \prime}$ northeast of the binary.

\section{DISCUSSION}

\subsection{Phase Offset}

The folded X-ray light curve appears to be offset from the ephemeris prediction by about 0.03 cycles, or $500 \mathrm{~s}$. The phase offset is greater than 0.006 cycles, or $100 \mathrm{~s}$, at $90 \%$ confidence level. Our check of the data reduction processes (such as the barycentric correction) has not revealed any potential systematics that could account for such a large offset. The linear ephemeris, taken from Zhang et al. (1995), has a nominal error of $11 \mathrm{~s}$ for this epoch. Moreover, their linear and quadratic ephemerides have diverged by less than $15 \mathrm{~s}$. Even though a long-term sinusoidal modulation in $O-C$ eclipse timings has been found (Patterson, Robinson, $\&$ Nather 1978), the magnitude of this effect is $70 \mathrm{~s}$. Thus, we believe that the phase offset likely does not result from an inaccurate ephemeris.

Instead, we consider it likely that the phase shift of the $\mathrm{X}$-ray light curve is caused by departure from axial symmetry of the DQ Her system. We see a considerable scatter in the times of the optical eclipse, up to $170 \mathrm{~s}$ in the $O-C$ timings, although some of it can be modeled by the sinusoidal term (see Fig. 4 of Zhang et al. 1995). Moreover, the eclipse light curves (see their Fig. 1) are obviously skewed, with ingress beginning around phase -0.08 and egress completing around phase 0.12 . This clearly demonstrates the departure from axisymmetry of the eclipsed object. We might still argue that the deepest point in the eclipse light curve marks the position of the unseen white dwarf. However, this is not necessarily the case, particularly if the eclipsed object is the back side of an asymmetric accretion disk (Petterson 1980). Interestingly, the radial velocity study of the secondary, using the $\mathrm{Na}$ I infrared doublet, shows that the inferior conjunction of the secondary is at phase $0.013 \pm 0.009$ on the eclipse ephemeris (Horne et al. 1993). This possible phase offset between the radial velocity curve of the secondary and the optical eclipse should be studied further. In any case, a nonaxisymmetric disk can result in a phase offset between the optical and X-ray eclipses.

Our best-fit X-ray eclipse width is also larger than the optical eclipse width, although in this case they can be equal within $90 \%$ confidence limits. We do not necessarily expect the two to be equal, since the model of Petterson (1980) for the phase shift of the $71 \mathrm{~s}$ oscillations suggests that the emission-weighted center of eclipsed light is somewhere on the back half of the accretion disk, not centered on the white dwarf. If true, the inclination angle $i$ derived with the latter assumption (Horne et al. 1993) would be an underestimate 
for a given $q$. We cannot solve this difficulty with the data in hand, so we treat this as an extra source of uncertainty in the analysis to follow.

\subsection{Wind-scattered $X$-Rays}

At this inclination, a partial eclipse is a signature of an extended emission region. Any X-ray emission or scattering from the surface of the accretion disk should be as deeply eclipsed as the optical light from the disk. Since the X-ray eclipse is much shallower than in the optical, we conclude that the X-rays must originate in a vertically extended region. Assuming the best-fit system parameters of Horne et al. (1993), we estimate that materials that are more than $0.28 a$ (where $a$ is the binary separation) above the orbital plane should remain uneclipsed. The limit could be somewhat larger if the inclination is underestimated by Horne et al. (1993).

Is this a single, extended X-ray source, or can there be an eclipsed component and an uneclipsed component? From the data in hand, we cannot rule out the latter possibility. We could test this if we had a higher quality X-ray light curve of the eclipse. A two-component model would predict a sharp ingress and egress, while a single extended source would result in a more gradual eclipse transition. Here we proceed assuming a single extended source, since we do not have a physical model corresponding to the two-component model.

The partial eclipse of UV emission lines in DQ Her also demonstrates the presence of an extended emission region (Córdova \& Mason 1985; Silber et al. 1996; Eracleous et al. 1998). Eracleous et al. (1998), in particular, have analyzed the time-resolved profile of the C IV $\lambda 1550$ line through the eclipse. At mideclipse, this line is clearly redshifted, which they argue is a strong indication that the residual line flux originates in an accretion disk wind (see also Fig. 7 of Córdova \& Mason 1985), even though an accretion disk wind is usually seen only in nonmagnetic CVs with high accretion rates (see, e.g., Drew \& Proga 2000). These systems have an accretion disk that extends all the way down to the white dwarf surface and an optically thick boundary layer. The presence of a wind in DQ Her, a system with no boundary layer and a luminous but truncated disk, suggests that the accretion geometry does not dictate whether a CV has a wind or not (Eracleous et al. 1998). DQ Her appears to be sufficiently luminous to drive a wind, since the fluxes of the He II recombination lines in DQ Her require an ionizing luminosity of the order of $10^{34} \mathrm{ergs} \mathrm{s}^{-1}$ (Silber et al. 1996; Eracleous et al. 1998).

The presence of the accretion disk wind in DQ Her might make this an unusual IP, since we are not aware of any reports of accretion disk winds in other IPs. Instead, it suggests a natural parallel with the dwarf nova OY Car in superoutburst and the nova-like system UX UMa. Both are bright nonmagnetic $\mathrm{CVs}$ with accretion disk wind; both are deeply eclipsing in the optical, yet no X-ray eclipses were observed in OY Car in superoutburst (Naylor et al. 1988; Pratt et al. 1999) or in UX UMa (Wood, Naylor, \& Marsh 1995). This is interpreted to imply that the central X-ray source in these systems cannot be seen directly, but instead is seen indirectly after scattering in an extended corona or wind. The EUVE observation of OY Car in superoutburst (Mauche \& Raymond 2000) confirms this basic picture, since the observed spectrum can be modeled as resonant scattering of continuum photons in an accretion disk wind. A wind, rather than a static corona, is strongly favored since the extreme-UV emission lines are seen to have a FWHM of $\sim 2300 \mathrm{~km} \mathrm{~s}^{-1}$. We believe the same resonant scattering model can be applied to the observed soft X-rays from DQ Her, except that the nature of the unseen central source must be different (see $\S 4.3$ ).

A static corona can also be the scattering site for the X-ray photons observed in DQ Her. However, that would make it different from OY Car, where the extreme-UV line widths prove the wind to be responsible for scattering. Also, the UV line profile during the eclipse is indicative of a wind, while we do not know if a static corona exists in this system. Finally, the depth of the partial eclipse demands a large vertical extent ( $>0.28 a$; see above) for the scattering region. This is well away from the disk and probably is an unlikely location for a static corona.

We therefore interpret the observed soft X-rays as being due to resonant scattering of an unseen central source. Furthermore, we prefer the accretion disk wind as the scattering site, pending final confirmation in the form of X-ray line widths. It is interesting to note that a partial X-ray eclipse is detected in DQ Her, while none has been found in OY Car or UX UMa. It is not clear, however, whether this indicates some difference in the wind geometry compared with DQ Her and nonmagnetic systems, or if this is simply due to the more extreme inclination angle in DQ Her.

\subsection{Nondetection of Reflected X-Rays}

The observed X-ray luminosity of $(3-5) \times 10^{30} \mathrm{ergs} \mathrm{s}^{-1}$ is likely to be a small fraction of the intrinsic luminosity of the central source, although this depends strongly on the unknown efficiency of scattering in the wind. The flux of the He II $\lambda 1640$ line has been used to estimate an ionizing luminosity of (1-2) $\times 10^{34} \mathrm{ergs} \mathrm{s}^{-1}$ (Silber et al. 1996; Eracleous et al. 1998).

Can this be in the form of a hard component $(k T \sim 20$ $\mathrm{keV}$ bremsstrahlung) usually seen in IPs? To investigate this question, we have modeled the reflection of such a component off the surface of the Roche lobe-filling secondary, applying the method of Still et al. (2001) developed for Her $\mathrm{X}-1$. X-rays hitting the cold material on the surface of the secondary can be scattered or absorbed; since softer photons are more likely to be absorbed, the scattered component is harder than the incident component. We have evaluated the 5-8 keV flux expected from reflection from the secondary as a function of the orbital phase, taking into account the shadowing by the accretion disk and the Roche lobe-filling shape of the secondary, assuming the system parameters of Horne et al. (1993), as listed in $\S 1$. For the computational details, see Still et al. (2001).

The reflection component should be most prominent at the highest energy band of Chandra, 5-8 keV. The viewing geometry for the reflection component is most favorable at orbital phase 0.5 ; it would result in a strong, sinusoidal orbital modulation. However, no such component is detected in our Chandra data in the 5-8 keV data. This can be converted to the upper limit of the bolometric luminosity of the hard component as a function of the disk thickness (Fig. 4). Taking our upper limit in the phase bin around phase $0.5,1 / 16$ cycle wide, to be $7.0 \times 10^{-4}$ counts s $^{-1}$, we estimate an upper limit to the $k T=20 \mathrm{keV}$ bremsstrahlung luminosity of $10^{33}$ ergs $\mathrm{s}^{-1}$ for a disk $H / R$ (height over radius) of less than 


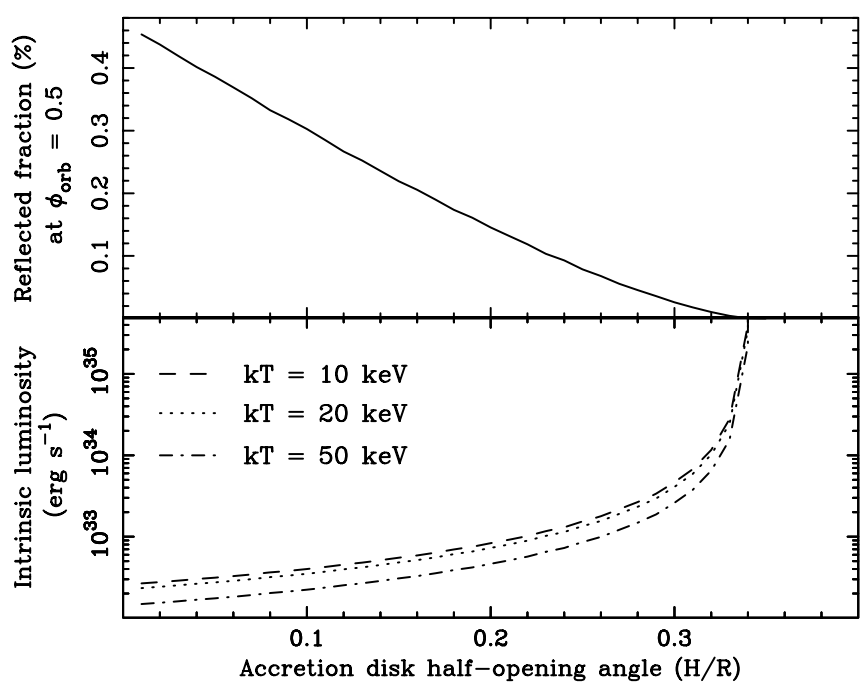

FIG. 4.-Top: Calculated efficiency of reflection from the secondary star at orbital phase 0.5 . The reflection fraction in the $5-8 \mathrm{keV}$ band is plotted as a function of the thickness of the accretion disk. Bottom: Inferred upper limit to the central hard component for three different bremsstrahlung temperatures, as a function of the disk thickness.

0.23 , or a half-opening angle smaller than $13^{\circ} .0\left(<14^{\circ} .6\right.$ for a $k T=50 \mathrm{keV}$ bremsstrahlung due to the different bolometric correction). If the intrinsic hard-component luminosity is $10^{34} \mathrm{ergs} \mathrm{s}^{-1}$, the reflected luminosity can be reduced to an undetectable level if the disk half-opening angle is greater than $17^{\circ} .7$ for $k T=20 \mathrm{keV}\left(>18^{\circ} .0\right.$ for $\left.k T=50 \mathrm{keV}\right)$, almost completely shadowing the secondary.

In comparison, the eclipsing and dipping low-mass X-ray binary EXO $0748-676$ has an inclination of $i \sim 75^{\circ}$ (Parmar et al. 1986). The presence of X-ray eclipses requires the opening angle of the disk to be less than $15^{\circ}$ toward the secondary in this system. Although azimuthal structures at such heights are commonly inferred (including one in U Gem $25^{\circ}$ above the disk; Szkody et al. 1996), we are not aware of claims for disks thicker than $15^{\circ}$ in the direction toward the secondary. We therefore conclude that the central hard X-ray component is less luminous than that inferred from the He II $\lambda 1640$ line, unless the disk in DQ Her has an unusually large thickness. Further studies of the disk thickness, however, are required to tighten the upper limit of the hard-component luminosity.

In contrast, the lack of a reflection component does not allow us to put a useful constraint on the central luminosity, if it has a soft spectrum. Accretion luminosity in DQ Her would be dominated by a soft, blackbody-like component, if the shock is buried within the white dwarf atmosphere. In addition, the entire photosphere of the primary may still be hot from the thermonuclear runaway of 1934 . The soft component in AM Her-type magnetic CVs has a typical temperature of 20-30 eV (Mauche 1999); even using a $k T=100 \mathrm{eV}$ blackbody, the spectrum is too soft to result in an appreciable reflection signature. Therefore, our results are consistent with the ionizing luminosity of $(1-2) \times 10^{34} \mathrm{ergs} \mathrm{s}^{-1}$ (Silber et al. 1996; Eracleous et al. 1998) if this is in the form of a soft component, regardless of the disk height.

\subsection{The Nova Shell around $D Q \mathrm{Her}$}

We appear to have detected extended emission features, one about $8^{\prime \prime}$ south-southwest of and another about $9^{\prime \prime}$ northeast of DQ Her. The latter location corresponds to a bright [N II] knot in the DQ Her shell, while the former has no obvious optical feature associated with it (Slavin, O'Brien, \& Dunlop 1995). We consider it likely that these small excesses are real and represent X-ray knots in the DQ Her shell. A similar, but much brighter, X-ray shell has been seen around GK Per (Nova Persei 1901; Balman \& Ögelman 1999). This shell has a soft thermal spectrum, with a prominent Ne Ix line at $0.9 \mathrm{keV}$ (Balman 2002). We do not have enough photons for a spectral analysis for the DQ Her shell, but it appears much softer than the GK Per shell. This could result from a different speed class (DQ Her was a slow nova, while GK Per was a fast nova), as well as from a different environment around DQ Her than around GK Per. The luminosity in the DQ Her knot appears to be a few times $10^{28} \mathrm{ergs} \mathrm{s}^{-1}$, subject to a large uncertainty in count rate-to-flux conversion because of the unknown spectral shape.

Given the probable discovery of X-rays from the shell and the apparent lack of strong hard X-rays from the central source, it may be useful to revisit the models of UV and optical spectra of the shell. For example, Ferland \& Truran (1981) assumed a hard X-ray-emitting magnetic CV with a luminosity in excess of $10^{34} \mathrm{ergs} \mathrm{s}^{-1}$ as the source of ionizing photons. Our work casts some doubt on such a picture. Petitjean, Boisson, \& Péquignot (1990), on the other hand, assumed a $10^{5} \mathrm{~K}$ blackbody source, which is consistent with our X-ray data of the central binary. However, how do the shell X-rays fit in?

Balman (2002) interprets the X-rays from the GK Per shell as a miniature supernova remnant. In this model, the nova ejecta are plowing into the surrounding interstellar medium and shock heated to X-ray-emitting temperatures. There certainly is ample kinetic energy in the nova ejecta to power shell X-ray emission, and the ejecta velocities are fast enough to result in X-ray-emitting temperatures. The photoionization model of UV and optical lines, on the other hand, suggests a cold shell. We regard this not as an outright contradiction, but as additional evidence for the coexistence of hot and cold matter in nova shells. We have long known of spectra that show both hot and cold components in nova shells (Payne-Gaposchkin 1957; Williams 1992). More recent imaging results (Slavin et al. 1995) and theoretical work (Lloyd, O'Brien, \& Bode 1997) show the importance of Rayleigh-Taylor instability. Nova shells are complex, multiphased entities, including, as it now appears, X-ray-emitting knots.

\section{CONCLUSIONS}

Our Chandra observation of the old nova DQ Her has revealed a partial X-ray eclipse. We believe that the most likely explanation is scattering in the accretion disk wind, with a central X-ray source that is hidden from our view by the accretion disk at all orbital phases. In this model, DQ Her is similar to OY Car in superoutburst and to UX UMa. With a higher signal-to-noise observation, such as we can hope to achieve with $X M M$-Newton, we should be able to measure the X-ray eclipse profile in detail and check the reality of the phase offset between X-ray and optical eclipses. We also detect an apparent weak extended feature, which we interpret as an X-ray-emitting knot in the nova shell. This would make DQ Her the second classical nova, after GK Per, with an X-ray-emitting shell. 


\section{REFERENCES}

Balman, S. 2002, in ASP Conf. Ser. 262, The High Energy Universe at Sharp Focus: Chandra Science, ed. E. M. Schlegel \& S. D. Vrtilek (San Francisco: ASP), 34

Balman, S., \& Ögelman, H. B. 1999, ApJ, 518, L111

Córdova, F. A., \& Mason, K. O. 1985, ApJ, 290, 67

Córdova, F. A., Mason, K. O., \& Nelson, J. E. 1981, ApJ, 245, 609

Downes, R. A., Webbink, R. F., Shara, M. M., Ritter, H., Kolb, U., \& Duerbeck, H. W. 2001, PASP, 113, 764

Drew, J. E., \& Proga, D. 2000, NewA Rev., 44, 21

Eracleous, M., Livio, M., Williams, R. E., Horne, K., Patterson, J., Martell, P., \& Korista, K. T. 1998, in ASP Conf. Ser. 137, Wild Stars in the Old West, ed. S. Howell, E. Kuulkers, \& C. Woodward (San Francisco: ASP), 438

Ferland, G. J., \& Truran, J. W. 1981, ApJ, 244, 1022

Herbig, G. H., \& Smak, J. I. 1992, Acta Astron., 42, 17

Horne, K. Welsh, W. F., \& Wade, R. A. 1993, ApJ, 410, 357

Liedahl, D. A., Osterheld, A. L., \& Goldstein, W. H. 1995, ApJ, 438, L115

Lloyd, H. M., O'Brien, T. J., \& Bode, M. F. 1997, MNRAS, 284, 137

Martin, P. G. 1989, in Classical Novae, ed. M. F. Bode \& A. Evans (New York: Wiley), 93

Mauche, C. W. 1999, in ASP Conf. Ser. 157, Annapolis Workshop on Magnetic Cataclysmic Variables, ed. C. Hellier \& K. Mukai (San Francisco: ASP), 157

Mauche, C. W. \& Raymond, J. C. 2000, ApJ, 541, 924

Mewe, R., Gronenschild, E. H. B. M., \& van den Oord, G. H. J. 1985, A\&AS, 62, 197

Mewe, R., Lemen, J. R., \& van den Oord, G. H. J. 1986, A\&AS, 65, 511
Naylor, T., Bath, G. T., Charles, P. A., Hassall, B. J. M., Sonneborn, G. van der Woerd, H., \& van Paradijs, J. 1988, MNRAS, 231, 237

Parmar, A. N., White, N. E., Giommi, P., \& Gottwald, M. 1986, ApJ, 308, 199

Patterson, J. 1994, PASP, 106, 209

Patterson, J., Robinson, E. L., \& Nather, R. E. 1978, ApJ, 224, 570

Payne-Gaposchkin, C. 1957, The Galactic Novae (Amsterdam: North Holland)

Petitjean, P., Boisson, C., \& Péquignot, D. 1990, A\&A, 240, 433

Petterson, J. A. 1980, ApJ, 241, 247

Pratt, G. W., Hassall, B. J. M., Naylor, T., Wood, J. H., \& Patterson, J. 1999, MNRAS, 309, 847

Shara, M. 1989, PASP, 101, 5

Silber, A. D., Anderson, S. F., Margon, B., \& Downes, R. A. 1996, ApJ, 462,428

Singh, K. P., Drake, S. A., Gotthelf, E. V., \& White, N. E. 1999, ApJ, 512, 874

Slavin, A. J., O’Brien, T. J., \& Dunlop, J. S. 1995, MNRAS, 276, 353

Still, M., et al. 2001, ApJ, 554, 352

Szkody, P.. Long, K. S., Sion, E. M., \& Raymond, J. C. 1996, ApJ, 469, 834

Walker, M. F. 1956, ApJ, 123, 68

Weisskopf, M. C., O’Dell, S. L., \& van Speybroeck, L. P. 1996, Proc. SPIE, 2805, 2

Williams, R. E. 1992, AJ, 104, 725

Wood, J. H., Naylor, T., \& Marsh, T. R. 1995, MNRAS, 274, 31

Zhang, E., Robinson, E. L., Stiening, R. F., \& Horne, K. 1995, ApJ, 454, 447 